\title{
Gradual Negotiations and Proportional Solutions
}

\author{
Shiran Rachmilevitch*
}

\begin{abstract}
I characterize the proportional $N$-person bargaining solutions by individual rationality, translation invariance, feasible set continuity, and a new axiominterim improvement. The latter says that if the disagreement point $d$ is known, but the feasible set is not-it may be either $S$ or $T$, where $S \subset T$ - then there exists a point $d^{\prime} \in S, d^{\prime}>d$, such that replacing $d$ with $d^{\prime}$ as the disagreement point would not change the final bargaining outcome, no matter which feasible set will be realized, $S$ or $T$. In words, if there is uncertainty regarding a possible expansion of the feasible set, the players can wait until it is resolved; in the meantime, they can find a Pareto improving interim outcome to commit to - a commitment that has no effect in case negotiations succeed, but promises higher disagreement payoffs to all in case negotiations fail prior to the resolution of uncertainty.
\end{abstract}

Keywords: Bargaining; Proportional solutions.

JEL Classification: C78; D74.

\section{Introduction}

I consider Nash's bargaining problem (Nash (1950)) and characterize the class of its proportional solutions. A bargaining problem is defined as a pair $(S, d)$ that satisfies

${ }^{*}$ Department of Economics, University of Haifa. 
the following:

- A1. $S \subset \mathbb{R}^{N}, d \in S$, and there exists an $x \in S$ such that $x>d ;^{1}$

- A2. $S$ is compact and comprehensive ${ }^{2}$

The set $S$, called the feasible set, consists of all the utility vectors that the players can achieve via cooperation; $d_{i}$ is player $i$ 's utility in the event that cooperation fails, hence $d$ is called the disagreement point. The collection of pairs $(S, d)$ that satisfy A1-A2 is denoted by $\mathcal{B}$; a solution is defined to be any function $\mu: \mathcal{B} \rightarrow \mathbb{R}^{N}$ that satisfies $\mu(S, d) \in S$ for all $(S, d) \in \mathcal{B}$. Given a vector $p>\mathbf{0},{ }^{3}$ let $\mu_{p}(S, d) \equiv d+\epsilon p$, where $\epsilon$ is the maximal number such that the expression on the right hand side is in $S$. A solution $\mu$ is proportional if there exists a vector $p>\mathbf{0}$ such that $\mu=\mu_{p}$. Proportional solutions were first characterized by Kalai (1977). The egalitarian solution, $E$, corresponds to the special case $p=\mathbf{1}$; i.e., $E \equiv \mu_{\mathbf{1}}$. The reader is referred to Thomson (1994) for an excellent discussion of the bargaining model.

I characterize the proportional solutions by the following axioms, in the statements of which $(S, d),\left(S^{\prime}, d^{\prime}\right)$, and $(T, e)$ are arbitrary elements of $\mathcal{B}$, and $\left(S^{n}, d\right)$ is an arbitrary sequence of elements of $\mathcal{B}$ sharing the same disagreement point.

\section{Individual Rationality (IR): $\mu(S, d) \geq d$.}

Translation Invariance (TINV): $\mu(S+t, d+t)=\mu(S, d)+t$ for all $t \in \mathbb{R}^{N}{ }^{4}$

\footnotetext{
${ }^{1}$ Vector inequalities: $x R y$ if and only if $x_{i} R y_{i}$ for all $i, R \in\{>, \geq\} ; x \supsetneqq y$ if and only if $x \geq y \&$ $x \neq y$.

${ }^{2}$ The set $S$ is comprehensive if for all $x, y \in S$ that satisfy $y \leq x$ it follows that $z \in S$, for every $z$ that satisfies $y \leq z \leq x$. It is strictly comprehensive if in addition $P(S) \equiv\{x \in S \mid y \supsetneqq x \Rightarrow y \notin$ $S\}=W P(S) \equiv\{x \in S \mid y>x \Rightarrow y \notin S\}$; that is, its strict and weak Pareto frontiers coincide. A bargaining problem whose $S$ is strictly comprehensive is a strictly comprehensive bargaining problem.

${ }^{3} \mathbf{0} \equiv(0, \cdots, 0)$. Similarly, $\mathbf{1} \equiv(1, \cdots, 1)$.

${ }^{4} S+t \equiv\{s+t \mid s \in S\}$.
} 
Feasible Set Continuity (S.CONT): If $S^{n}$ converges to $S$ in the Hausdorff topology and $(S, d) \in \mathcal{B}$, then $\mu(S, d)=\lim _{n} \mu\left(S^{n}, d\right)$.

Interim Improvement (II): If $d=e \equiv d^{*}$ and $S \subset T$, then there exists a $d^{\prime}>d^{*}$, such that $\left(S, d^{\prime}\right),\left(T, d^{\prime}\right) \in \mathcal{B}, \mu\left(S, d^{\prime}\right)=\mu\left(S, d^{*}\right)$, and $\mu\left(T, d^{\prime}\right)=\mu\left(T, d^{*}\right)$.

The first two axioms are very weak; they are satisfied by all the major solutions considered in the literature. S.CONT is satisfied by all the continuous ones, and the major solutions in the literature are continuous. The fourth axiom, II, captures the following idea. Suppose that the disagreement point $d$ is known with certainty, but the feasible set is not-it may be either $S$ or $T$, where $S \subset T$. That is, it is known with certainty that all the options in $S$ are feasible, but there are additional options, those in $T \backslash S$, the feasibility of which is uncertain. A natural course of action in this case is to "wait and see": once the uncertainty is resolved, the relevant bargaining outcome will be implemented. However, since some time passes before the realization of uncertainty, the players face a risk, as during this time negotiations may break down, an event in which they end up with the low payoffs $d$. A natural way to insure themselves against such an event is by signing an intermediate binding contact that specifies the payoffs in case that the bargaining procedure breaks down prior to the resolution of uncertainty. This, however, may be a difficult task, because if the interim contract affects the final outcome, then the players may behave strategically, and as a result may prefer not to sign such a contract at all. The axiom II guarantees that this is never the case: one can always find a point $d^{\prime} \in S$ with $d^{\prime}>d$, such that replacing $d$ with $d^{\prime}$ as the disagreement point would not change the final bargaining outcome, independent of the realization of uncertainty. ${ }^{5}$

\footnotetext{
${ }^{5}$ Axioms concerning uncertainty regarding components of the bargaining problem have been studied extensively in the literature. The two most notable examples are Perles and Maschler's super additivity (see Perles and Maschler (1981)) and Chun and Thomson's disagreement point concavity (see Chun and Thomson (1990)). Holding the disagreement point $d$ fixed, the former requires a
} 
Note that the definition of II allows for $S=T$. That is, II is a necessary condition for a "step-by-step" bargaining process, where the players work their way from the disagreement point to the final outcome via a sequence of intermediate agreements (though there is nothing in II to guarantee that this process is finite). Kalai (1977) introduced the "step-by-step" idea and applied it to obtain one of the first characterizations of the proportional solutions. Formally, he considered the following axiom, in the statement of which $(S, d)$ and $(T, e)$ are arbitrary elements of $\mathcal{B}$.

Step by Step Negotiations (SSN): If $d=e \equiv d^{*}, S \subset T$, and $\left(T, \mu\left(S, d^{*}\right)\right) \in \mathcal{B}$, then $\mu\left(T, d^{*}\right)=\mu\left(T, \mu\left(S, d^{*}\right)\right)$.

In words, SSN says that whenever the players face two nested problems with a common disagreement point, they can first solve the smaller (and presumably simpler) problem, and then regard its solution as the disagreement point of the "continuation problem." In SSN the steps along the bargaining process correspond to nested feasible sets; in II, by contrast, they correspond to intermediate disagreement points.

The rest of the paper consists of two sections: Section 2 contains the main result and related analysis, and Section 3 focuses on comparing II and SSN.

mixture of two feasible sets, $S$ and $T$, to lead to a solution point that lies above the respective mixture of the solutions of $(S, d)$ and $(T, d)$; the latter imposes the analogous requirement on mixtures of two disagreement points, when the feasible set is fixed. It is worth noting that as opposed to these axioms, II does not assume commonly known probabilities. That is, it accommodates the case where player $i$ believes that the options in $T \backslash S$ will become available with probability $p_{i}$, where $p_{i}$ is an idiosyncratic (and not necessarily commonly known) value. Moreover, II does not even assume that such a $p_{i}$ exists. 


\section{The main result}

Consider the following axioms, in the statements of which $(S, d)$ and $(T, e)$ are arbitrary elements of $\mathcal{B}$, and $\left(S, d_{n}\right)$ is an arbitrary sequence of elements of $\mathcal{B}$ sharing the same feasible set.

Weak Pareto Optimality (W.PO): $\mu(S, d) \in W P(S) .{ }^{6}$

Weak Disagreement Point Continuity (W.D.CONT): If $\mu\left(S, d_{n}\right)=x$ for all $n$, $d_{n} \rightarrow d$ and $(S, d) \in \mathcal{B}$, then $\mu(S, d)=x$.

Strong Individual Rationality (S.IR): $\mu(S, d)>d$.

Independence of Non Individually Rational Alternatives (INIR): $\mu(S, d)=$ $\mu\left(S_{d}, d\right)$, where $S_{d} \equiv\{x \in S \mid x \geq d\}$.

Monotonicity (MON): If $d=e \equiv d^{*}$ and $S \subset T$, then $\mu\left(S, d^{*}\right) \leq \mu\left(T, d^{*}\right)$.

The first axiom in the list is obvious; the second axiom is a weaker version of the more common disagreement point continuity, which makes the same requirement, but without the restriction to sequences of elements that give rise to the same solution point; the third and fourth axioms, which are due to Roth (1977) and Peters (1986) respectively, strengthen IR; the fifth axiom, which is due to Kalai (1977), says that if more options become available, no one should get hurt.

Lemma 1. S.CONT and TINV imply W.D.CONT.

Proof. Let $\mu$ be a solution that satisfies S.CONT and TINV. Suppose that $\left(S, d_{n}\right) \in \mathcal{B}$

\footnotetext{
${ }^{6} \mathrm{~A}$ natural strengthening of this axiom is Pareto Optimality (PO), which requires $\mu(S, d) \in P(S)$ for all $(S, d) \in \mathcal{B}$.
} 
and $\mu\left(S, d_{n}\right)=x$ for all $n, d_{n} \rightarrow d$ and $(S, d) \in \mathcal{B}$. By TINV, $\mu\left(S-d_{n}, \mathbf{0}\right)=x-d_{n}$. By S.CONT, $\mu(S-d, \mathbf{0})=x-d$. Applying TINV again gives $\mu(S, d)=x$.

Lemma 2. II, IR, TINV, and S.CONT imply SSN.

Proof. Let $\mu$ be a solution that satisfies II, IR, TINV, and S.CONT. By the latter axiom, it suffices to prove that SSN holds on the domain of strictly comprehensive problems. Let $(S, d),\left(T, d^{\prime}\right) \in \mathcal{B}$ be such that $d^{\prime}=d, S \subset T$ and are both strictly comprehensive, and $(T, \mu(S, d)) \in \mathcal{B}$. By Lemma $1 \mu$ satisfies W.D.CONT. By II and W.D.CONT, it follows that there exists a sequence $\left\{d_{n}\right\} \subset S$, such that $\mu\left(S, d_{n}\right)=$ $\mu(S, d)$ and $\mu\left(T, d_{n}\right)=\mu(T, d)$ for all $n$, and $d^{*} \equiv \lim _{n \rightarrow \infty} d_{n} \in W P(S) .{ }^{7}$ Since $S$ is strictly comprehensive, $d^{*} \in P(S)$. By IR $d_{n} \leq \mu\left(S, d_{n}\right)=\mu(S, d)$, hence $d^{*} \leq \mu(S, d)$. Since $d^{*} \in P(S), d^{*}=\mu(S, d)$. Since $(T, \mu(S, d))=\left(T, d^{*}\right) \in \mathcal{B}$, and $\mu\left(T, d_{n}\right)=\mu(T, d)$ for all $n$, W.D.CONT implies that $\mu(T, \mu(S, d))=\mu\left(T, d^{*}\right)=$ $\mu(T, d)$.

Lemma 3. II, IR, TINV, and S.CONT imply WPO.

Proof. Let $\mu$ be a solution that satisfies II, IR, TINV and S.CONT, and let $(S, d) \in \mathcal{B}$. By the argument from Lemma 2 there exists a sequence $\left\{d_{n}\right\}$ such that $\mu\left(S, d_{n}\right)=$ $\mu(S, d)$ for all $n$, that converges to a limit $d^{*} \in W P(S)$. By IR, $d_{n} \leq \mu\left(S, d_{n}\right)=$ $\mu(S, d)$, therefore $d^{*} \leq \mu(S, d)$. Therefore, $\mu(S, d) \in W P(S)$.

Lemma 4. II, IR, TINV, and S.CONT imply INIR.

Proof. Let $\mu$ be a solution that satisfies II, IR, TINV, and S.CONT, and let $(S, d) \in \mathcal{B}$. By S.CONT we may assume that $S$ is strictly comprehensive. Assume by contradiction that $x \equiv \mu(S, d) \neq \mu\left(S_{d}, d\right) \equiv y$. By Lemma 3 and the fact that $S$ is strictly

\footnotetext{
${ }^{7}$ Existence of the limit follows from the compactness of $S_{d}$. Let $L$ be the set of all limits of these sequences. By the aforementioned existence argument, $L \neq \emptyset$. Define the binary relation $\succ$ on $L$ by: $l \succ l^{\prime}$ if and only if $l_{1}>l_{1}^{\prime}$. Since $L \subset S_{d}$ and the latter is compact, there exists a $\succ$ maximal element in the closure of $L, l^{*}$, which belongs to $S_{d}$. I argue that $l^{*} \in W P(S)$. Otherwise $\left(S, l^{*}\right) \in \mathcal{B}$, and therefore, by II, there exists an $l^{\prime}>l^{*}$ such that $\left(S, l^{\prime}\right) \in \mathcal{B}, \mu\left(S, l^{\prime}\right)=\mu\left(S, l^{*}\right)$, and $\mu\left(T, l^{\prime}\right)=\mu\left(T, l^{*}\right)$, in contradicts to the $\succ$-maximality of $l^{*}$.
} 
comprehensive, $x$ and $y$ are two distinct points of $P(S)$. Now, we can find $\lambda<\lambda^{\prime}<1$, both sufficiently close to 1 , such that $x \notin Q_{z}$, where $Q \equiv \lambda^{\prime} S_{d}$ and $z \equiv \lambda y{ }^{8,9}$ This contradicts the fact that $\mu$ satisfies SSN and IR.

Given $r>0$, let $\Delta_{r} \equiv\left\{x \in \mathbb{R}_{+}^{N} \mid \sum_{i=1}^{N} x_{i} \leq r\right\}$.

Lemma 5. Let $\mu$ be a solution that satisfies II, IR, TINV, and S.CONT. Then $\mu\left(\Delta_{k r}, \mathbf{0}\right)=k \mu\left(\Delta_{r}, \mathbf{0}\right)$ for all $r>0$ and $k \in \mathbb{N}$.

Proof. Make the assumptions of the lemma and let $r>0$. The lemma is obvious for $k=1$. I will prove that it holds for $k \geq 2$ by induction.

Consider $k=2$. Here, we need to prove $\mu\left(\Delta_{2 r}, \mathbf{0}\right)=2 \mu\left(\Delta_{r}, \mathbf{0}\right)$. By SSN and TINV, the left hand side equals $\mu\left(\Delta_{2 r}, \mu\left(\Delta_{r}, \mathbf{0}\right)\right)=\mu\left(\Delta_{2 r}-\mu\left(\Delta_{r}, \mathbf{0}\right), \mathbf{0}\right)+\mu\left(\Delta_{r}, \mathbf{0}\right)$; therefore, we need to prove $\mu\left(\Delta_{2 r}-\mu\left(\Delta_{r}, \mathbf{0}\right), \mathbf{0}\right)=\mu\left(\Delta_{r}, \mathbf{0}\right)$. By INIR, the left hand side equals $\mu\left(\left(\Delta_{2 r}-\mu\left(\Delta_{r}, \mathbf{0}\right)\right) \cap \mathbb{R}_{+}^{N}, \mathbf{0}\right)$; finally, since $\mu$ satisfies WPO, $\left(\Delta_{2 r}-\mu\left(\Delta_{r}, \mathbf{0}\right)\right) \cap \mathbb{R}_{+}^{N}=\Delta_{r}$. Therefore, the lemma is true for $k=2$.

The inductive step: Suppose that the lemma is true for $(k-1)$, where $k \geq 2$. By SSN and TINV,

$$
\mu\left(\Delta_{k r}, \mathbf{0}\right)=\mu\left(\Delta_{k r}-\mu\left(\Delta_{(k-1) r}, \mathbf{0}\right), \mathbf{0}\right)+\mu\left(\Delta_{(k-1) r}, \mathbf{0}\right) .
$$

By the induction's hypothesis $\mu\left(\Delta_{(k-1) r}, \mathbf{0}\right)=(k-1) \mu\left(\Delta_{r}, \mathbf{0}\right)$. Therefore, we need to prove that $\mu\left(\Delta_{k r}-\mu\left(\Delta_{(k-1) r}, \mathbf{0}\right), \mathbf{0}\right)=\mu\left(\Delta_{r}, \mathbf{0}\right)$. By INIR, the left hand side equals $\mu\left(\left(\Delta_{k r}-\mu\left(\Delta_{(k-1) r}, \mathbf{0}\right)\right) \cap \mathbb{R}_{+}^{N}, \mathbf{0}\right)$; by WPO, $\left(\Delta_{k r}-\mu\left(\Delta_{(k-1) r}, \mathbf{0}\right)\right) \cap \mathbb{R}_{+}^{N}=\Delta_{r}$.

Lemma 6. II, IR, TINV, and S.CONT imply MON.

Proof. Let $\mu$ be a solution that satisfies II, IR, TINV, and S.CONT. By the latter, it suffices to prove that MON holds for strictly comprehensive problems. Let then

\footnotetext{
${ }^{8} \lambda S \equiv\{\lambda s \mid s \in S\}$.
}

${ }^{9}$ The strict comprehensiveness of $S$ guarantees that $(Q, \lambda y) \in \mathcal{B}$. 
$(S, d),(T, d) \in \mathcal{B}$ be two strictly comprehensive problems such that $S \subset T$. Assume by contradiction that there is some $i$ such that $x_{i}>y_{i}$, where $x \equiv \mu(S, d)$ and $y \equiv \mu(T, d)$. By the arguments from Lemma 2 there exists a sequence $\left\{d_{n}\right\} \subset S$ such that $\mu\left(S, d_{n}\right)=\mu(S, d)$ and $\mu\left(T, d_{n}\right)=\mu(T, d)$ for all $n$, and $d^{*} \equiv \lim _{n \rightarrow \infty} d_{n} \in$ $W P(S)=P(S)$. By IR $d_{n} \leq x$ for all $x$, hence $d^{*} \leq x$; therefore, $d^{*}=x$. By Lemma $1 \mu$ satisfies W.D.CONT, and therefore $y=\mu(T, d)=\mu\left(T, d^{*}\right)=\mu(T, x)$. However, $x_{i}>y_{i}$ contradicts IR.

Let $p(r) \equiv \mu\left(\Delta_{r}, \mathbf{0}\right)$.

Lemma 7. Let $\mu$ be a solution that satisfies II, IR, TINV, and S.CONT. Then $p($. is homogeneous of degree one. That is, $p(r)=\operatorname{rp}(1)$ for every $r>0$.

Proof. Let $\mu$ be a solution that satisfies II, IR, TINV, and S.CONT, and let $r>0$. Clearly there is nothing to prove if $r=1$, so consider $r \neq 1$. Suppose first that $r \leq \frac{1}{2}$. For every integer $n$ there exists a unique integer $k$ such that $k\left(\frac{1}{2}\right)^{n} \leq r \leq(k+1)\left(\frac{1}{2}\right)^{n}$. If there is an $n$ such that one of these inequality is satisfied as equality, then, by Lemma 5, we are done. Suppose, on the other hand, that $k\left(\frac{1}{2}\right)^{n}<r<(k+1)\left(\frac{1}{2}\right)^{n}$ for every $n$. Let $S_{n} \equiv \Delta_{k\left(\frac{1}{2}\right)^{n}}$ and $S_{n}^{+} \equiv \Delta_{(k+1)\left(\frac{1}{2}\right)^{n}}$. By MON,

$$
\mu\left(S_{n}, \mathbf{0}\right) \leq p(r) \leq \mu\left(S_{n}^{+}, \mathbf{0}\right) .
$$

Note that $\mu\left(S_{n}, \mathbf{0}\right)=p\left(k\left(\frac{1}{2}\right)^{n}\right)=k p\left(\left(\frac{1}{2}\right)^{n}\right)=k \mu\left(\Delta_{\left(\frac{1}{2}\right)^{n}}, \mathbf{0}\right)$; the first and third equalities follow from the definition of $p$, and the second also involves Lemma 5. Also, $p(1)=\mu\left(\Delta_{1}, \mathbf{0}\right)=\mu\left(\Delta_{2^{n}\left(\frac{1}{2}\right)^{n}}, \mathbf{0}\right)=2^{n} \mu\left(\Delta_{\left(\frac{1}{2}\right)^{n}}, \mathbf{0}\right)$ follows from Lemma 5. Therefore, $\mu\left(\Delta_{\left(\frac{1}{2}\right)^{n}}, \mathbf{0}\right)=\left(\frac{1}{2}\right)^{n} p(1)$ for every $n$. Therefore, $\mu\left(S_{n}, \mathbf{0}\right)=k\left(\frac{1}{2}\right)^{n} p(1)$. Similarly, $\mu\left(S_{n}^{+}, \mathbf{0}\right)=(k+1)\left(\frac{1}{2}\right)^{n} p(1)$. Plugging this into (1) gives $k\left(\frac{1}{2}\right)^{n} p(1) \leq p(r) \leq$ $(k+1)\left(\frac{1}{2}\right)^{n} p(1)$. Taking $n \rightarrow \infty$ gives $r p(1) \leq p(r) \leq r p(1)$, so $p(r)=r p(1)$. Similar arguments cover the case $r>\frac{1}{2}$.

Given $r>0$, let $C(r) \equiv\left\{x \in \mathbb{R}_{+}^{N} \mid x \leq p(r)\right\}$. 
Lemma 8. Let $\mu$ be a solution that satisfies II, IR, TINV, and S.CONT, and let $r>0$. Then $\mu(C(r), \boldsymbol{O})=p(r)$.

Proof. Let $\mu$ be a solution that satisfies II, IR, TINV, and S.CONT, and let $r>0$. Assume by contradiction that $x \neq p(r)$, where $x \equiv \mu(C(r), \mathbf{0})$. By SSN, $p(r)=$ $\mu\left(\Delta_{r}, x\right)$. By II there exists a $y>x$ such that $\left(\Delta_{r}, y\right) \in \mathcal{B}$ and $\mu\left(\Delta_{r}, y\right)=p(r)$. Therefore, by IR, $p(r) \geq y>x$, in contradiction to WPO.

Theorem 1. A solution satisfies IR, TINV, S.CONT, and II, if and only if it is proportional.

Proof. It is clear that every proportional solution satisfies the aforementioned axioms. Conversely, let $\mu$ be an arbitrary solution satisfying them. I will prove that $\mu=\mu_{p}$, where $p \equiv p(1){ }^{10}$

By S.CONT and TINV, it is enough to prove that $\mu$ coincides with $\mu_{p}$ on the the class of strictly comprehensive bargaining problems with disagreement point $\mathbf{0}$. Let then $(S, \mathbf{0})$ be such a problem. Let $r>0$ be the unique number such that $r p(1)=\mu_{p}(S, \mathbf{0})$. Assume by contradiction that $\mu(S, \mathbf{0}) \neq \mu_{p}(S, \mathbf{0})$. Since $\mu_{p}(S, \mathbf{0}) \in$ $W P(S)=P(S)$, there exists an $i$ such that $\mu_{i}(S, \mathbf{0})<\mu_{p, i}(S, \mathbf{0})=r p_{i}(1)$. Let $r^{\prime}<r$ be such that

$$
\mu_{i}(S, \mathbf{0})<r^{\prime} p_{i}(1)
$$

We have that $\mu(S, \mathbf{0})=\mu\left(S, \mu\left(C\left(r^{\prime}\right), \mathbf{0}\right)\right) \geq \mu\left(C\left(r^{\prime}\right), \mathbf{0}\right)=p\left(r^{\prime}\right)=r^{\prime} p(1)$; the first equality is by SSN, the inequality is by IR, and the following equalities are by Lemma 8 and Lemma 7, respectively. This, of course, contradicts (2).

\subsection{Independence of the axioms}

Below are four solutions, each of which violates exactly one of the axioms listed in Theorem 1 and satisfies the remaining three.

\footnotetext{
${ }^{10} p(1)>\mathbf{0}$ follows from II and IR.
} 
1. All but IR: Consider $-E(S, d) \equiv d-x \cdot \mathbf{1}$, where $x$ is the maximal number such that the right hand side is in $S$.

2. All but II: The Nash solution (Nash (1950)). This solution assigns to each $(S, d) \in \mathcal{B}$ the maximizer of $\Pi_{i}\left(x_{i}-d_{i}\right)$ over $S_{d} \cdot{ }^{11}$

3. All but TINV (for $N=2$ ): Fix an $\varepsilon>0$. Let $D \equiv\left\{x \in \mathbb{R}^{2} \mid x_{1}=x_{2}\right\}$. Denote by $B$ the open band of width $\varepsilon$ around the plane's diagonal $D$; i.e. $B \equiv\left\{x \in \mathbb{R}^{2}\|\mid x-D\|<\right.$ $\varepsilon\}$. Let $\hat{\mu}$ be the following solution:

$$
\hat{\mu}(S, d) \equiv \begin{cases}E(S, d) & \text { if } d \in B \\ \mu_{(2,1)}(S, d) & \text { if } d \notin B \text { and } d_{1}>d_{2} \\ \mu_{(1,2)}(S, d) & \text { if } d \notin B \text { and } d_{1}<d_{2}\end{cases}
$$

It is easy to see that $\hat{\mu}$ satisfies all the axioms of Theorem 1 besides TINV. This solution captures the idea that the bargaining outcome may be sensitive to the outside options: if the outside options are sufficiently close, then the solution splits the surplus equally; otherwise, the stronger player is being favored in terms of receiving a larger proportion of the surplus.

4. All but S.CONT. Let $N=2$ and consider the lexicographic extension of $E, E_{l e x}$, which is defined as follows. ${ }^{12}$ For every $(S, d) \in \mathcal{B}$ there exists at most one player $i$ such that the $i$-th coordinate of $E(S, d)$ can be increased without decreasing coordi-

\footnotetext{
${ }^{11}$ Besides the proportional solutions, all the prominent solutions considered in the literature violate II (which is an obvious corollary of Theorem 1, as besides II the remaining axioms are very weak). Examples for such solutions include the Kalai-Smorodinsky solution (Kalai and Smorodinsky (1975)), the Perles-Maschler solution (Perles and Maschler (1981)), the equal sacrifice solution (Aumann and Maschler (1985), Chun (1988)), and the equal area solution (Anbarci (1993), Anbarci and Bigelow (1994)).

${ }^{12}$ The generalization to $N>2$ is straightforward. This solution has been studied by Chun (1989). The lexicographic extension of any other proportional solution $\mu_{p}$ is defined analogously.
} 
nate $j \neq i$ and without leaving $S ; E_{\text {lex }}$ is obtained by applying the maximal payoff increase to this $i$ if such an $i$ exists, and $E_{l e x}=E$ otherwise. It is easy to see that this solution satisfies all the axioms besides S.CONT.

Example 4 may lead one to suspect that once S.CONT is deleted from the axiom list in Theorem 1, a characterization of the lexicographic extensions of the proportional solutions is obtained. This is not true; in fact, this is not true even if PO is added to the axiom list. I conclude this subsection with an example of a 2-person solution, which is not proportional, and that satisfies PO, IR, TINV, and II. Let $S_{0}=\{x \in$ $\left.\mathbb{R}_{+}^{2} \mid x_{1}^{2}+x_{2}^{2} \leq 1\right\}$ and consider the following solution,

$\tilde{\mu}(S, d) \equiv \begin{cases}\left(\sqrt{\frac{1}{2}}, \sqrt{\frac{1}{2}}\right)+t & \text { if there is a } t \text { such that } S=S_{0}+t \text { and } d<\left(\sqrt{\frac{1}{2}}, \sqrt{\frac{1}{2}}\right)+t \\ E_{\text {lex }}(S, d) & \text { otherwise }\end{cases}$

\subsection{Other domains}

The feasible sets are allowed to be non-convex. The are many reasons why nonconvexities may arise in bargaining. For example, the players may not have access to a randomization device, and even if they do they may prefer not to use it, because they may view some issues as too important to be decided on by gambling. However, all the analysis above remains equally valid if convexity is added (to A1-A2 above). In other words, none of the arguments above invokes non-convex feasible sets.

Similarly, the analysis remains valid if compactness is replaced by unboundedness from below, ${ }^{13}$ coupled with compactness of $S_{d}$. Namely, if free disposal of utility is allowed. The only thing that needs to be changed is Example 1, since the solution it describes is not well-defined when the feasible set is unbounded from below. The modification $-\tilde{E}(S, d) \equiv E(S, d)-\mathbf{1}$ does the job.

\footnotetext{
${ }^{13}$ i.e., $x \in S$ and $y \leq x$ implies $y \in S$.
} 


\subsection{Homogeneity}

As seen above, MON follows quite straightforwardly from the four axioms of Theorem 1. Since Kalai (1977) derived, in a relatively short proof, a characterization of the proportional solutions in which MON is the central axiom, ${ }^{14}$ one may suspect that some of the steps (lemmas) derived above are superfluous. This is not the case, because Kalai's theorem, as opposed to Theorem 1, also assumes the following axiom. In the axiom's statement, as usual, $(S, d)$ is an arbitrary element of $\mathcal{B}$.

Homogeneity (HOM): $\mu(\lambda S, \lambda d)=\lambda \mu(S, d)$ for all $\lambda>0$.

In fact, a significance part of the analysis above is dedicated to establishing HOM on a restricted domain of problems - the domain of problems $(S, d)$ where $S$ is a simplex. Together with the other axioms, homogeneity on this restricted domain turns out to be sufficient for proportionality.

Apart from being a technicality, dispensing with HOM has a conceptual appeal. Suppose that the bargaining problem is solved by an arbitrator, who recommends the players an outcome. This arbitrator may have certain objectives, or guidelines, in mind. For example, he may seek to promote fairness and efficiency. Since the "right" balance between them may depend on the bargaining problem itself-in particular, it may depend on the size of the "surplus" that is to be divided - the solution he employs need not satisfy HOM.

To see this point more formally, consider the following subdomain of problems. Let $\mathcal{B}^{*} \subset \mathcal{B}$ consist of those $(S, d) \in \mathcal{B}$ such that $S$ and is convex, strictly comprehensive, and satisfies the following: for all distinct $x, y \in P(S)$ and $\lambda \in(0,1), \lambda x+(1-\lambda) y \notin$ $P(S)$. In words, $\mathcal{B}^{*}$ is the class of smooth and convex bargaining problems. The

\footnotetext{
${ }^{14}$ In his 1977 paper, Kalai derived the first characterizations of the proportional solutions. The first theorem in his paper utilizes MON as the main axiom, and the second theorem in his paper utilizes SSN.
} 
utilitarian solution is well defined on this class. For each $(S, d) \in \mathcal{B}^{*}$, its utilitarian solution, denoted $U(S, d)$, is the unique point of $S_{d}$ that maximizes the sum of the players' utilities. Given a number $t>0$, consider the following solution on $\mathcal{B}^{*}:{ }^{15}$

$$
\mu^{t}(S, d) \equiv \begin{cases}E(S, d) & \text { if } d+\frac{t}{N} \cdot \mathbf{1} \notin \operatorname{int} S \\ U\left(\left\{x \in S \mid x \geq d+\frac{t}{N} \cdot \mathbf{1}\right\}, d+\frac{t}{N} \cdot \mathbf{1}\right) & \text { otherwise }\end{cases}
$$

This solution guarantees to all players the minimal utility gain $\frac{t}{N}$ whenever possible, and distributes any further surplus efficiently. In those problems where guaranteeing the per-capita gain $\frac{t}{N}$ is impossible, gains are distributed equally. This is a sensible rationale for solving bargaining situations; in particular-for an arbitrator who is interested in efficiency, but only as long as a "minimal degree of egalitarianism" is maintained. Obviously, this solution violates HOM.

\subsection{Relaxing II}

The axiom II involves a strict inequality: it requires that the interim improvement $d^{\prime}$ satisfies $d^{\prime}>d^{*}$. Since weak inequalities are more common in bargaining theory (and, it is probably safe to say, in economic theory at large), one may want to weaken II to demand only $d^{\prime} \supsetneqq d^{*}$. Call the resulting axiom weak interim improvement (W.II). One can verify that replacing II by W.II in Theorem 1 results in a characterization of all the solutions of the form $\mu_{p}$ where $p \supsetneqq \mathbf{0}$ (instead of $p>\mathbf{0}$ ). In particular, as opposed to II, W.II is consistent with dictatorial solutions. Formally, the $i$-th dictatorial solution, $D_{i}$, is given by $D_{i}(S, d) \equiv\left(x, d_{-i}\right)$, where $x$ is the maximal number such that the right hand side is in $S$. It is easy to see that $D_{i}$ satisfies W.II but not II.

Moreover, II, as opposed to W.II, expresses interpersonal utility comparisons. More formally, a solution does not allow for such comparisons if it satisfies the fol-

\footnotetext{
${ }^{15}$ int $S$ is a shorthand to the "interior of $S . "$
} 
lowing axiom, in the statement of which $(S, d)$ is an arbitrary element of $\mathcal{B}$.

Independence of Equivalent Utility Representations (IEUR): If $\lambda: \mathbb{R}^{N} \rightarrow \mathbb{R}^{N}$ is a positive affine transformation, then $\mu(\lambda \circ S, \lambda \circ d)=\lambda \circ \mu(S, d) \cdot{ }^{16,17}$

The fact that W.II is consistent with IEUR is obvious, because the dictatorial solutions satisfy both. The fact that II is inconsistent with IEUR is proved below; for the sake of simplicity and ease of exposition only, I prove this inconsistency for $N=2$.

Proposition 1. Let $N=2$. Then, there does not exist a solution that satisfies II and IEUR.

Proof. Let $N=2$. Assume by contradiction that there exists a solution that satisfies II and IEUR. Let $R=\left\{x \in \mathbb{R}_{+}^{2} \mid x \leq(1,1)\right\}$ and let $R^{\prime}=\left\{x \in \mathbb{R}_{+}^{2} \mid x \leq(2,1)\right\}$. By II, there exists a point $(a, b)>\mathbf{0}$, such that replacing $\mathbf{0}$ with $(a, b)$ will not change the outcome in either of the problems $(R, \mathbf{0})$ and $\left(R^{\prime}, \mathbf{0}\right)$. Applying IEUR to the problems $(R, \mathbf{0})$ and $(R,(a, b))$, we see that $(a, b)$ must satisfy $\frac{1-b}{1-a}=1$. Applying the same argument to $\left(R^{\prime}, \mathbf{0}\right)$ and $\left(R^{\prime},(a, b)\right)$, we conclude that $\frac{1-b}{1-a}=2$, a contradiction.

The discussion above may lead one to suspect that the combination of W.II, IR, and IEUR results in a characterization of the dictatorial solutions. As the following 2-person example shows, this is not true.

$\mu^{*}(S, d) \equiv \begin{cases}x & \text { if } x \text { is the unique kink in the relative interior of } W P\left(S_{d}\right) \\ D_{1}(S, d) & \text { otherwise }\end{cases}$

\footnotetext{
${ }^{16} \lambda=\left(\lambda_{1}, \cdots, \lambda_{N}\right)$ is a positive affine transformation if the following holds for each $i: \lambda_{i}(x)=$ $\alpha_{i} x+\beta_{i}$, where $\alpha_{i}>0$.

${ }^{17} \mathrm{TINV}$ is obtained as a special case of this axiom, where $\alpha_{1}=\alpha_{2}=\cdots=\alpha_{N}=1$.
} 


\section{II vs. SSN}

II and SSN share a similar flavor: both describe a "graduality" property. They are, however, logically incomparable.

Proposition 2. SSN and II are logically incomparable.

Proof. Consider the disagreement solution $\mu(S, d) \equiv d$. It is immediate that it satisfies SSN and violates II. The solution $E_{l e x}$ satisfies II but not SSN.

As the following proposition shows, the fact that S.IR is violated in the example of a solution that satisfies SSN but violates II is not coincidental.

Proposition 3. SSN and S.IR imply II.

Proof. Let $\mu$ satisfy S.IR and SSN and let $(S, d)$ and $(T, d)$ be two elements of $\mathcal{B}$ with $S \subset T$, who share the disagreement point $d$. We need to find a $d^{\prime}>d$ such that $\left(S, d^{\prime}\right) \in \mathcal{B}$ and $\mu\left(X, d^{\prime}\right)=\mu(X, d)$ for both $X \in\{S, T\}$. Clearly we can pick a subset $Q \subset S$ such that $(Q, d) \in \mathcal{B}$ and $W P(Q) \cap W P(S)=\emptyset$. Let $d^{\prime} \equiv \mu(Q, d)$. By S.IR, $d^{\prime}>d$. By SSN, $\mu\left(X, d^{\prime}\right)=\mu(X, d)$ for both $X \in\{S, T\}$.

Proposition 3 can be viewed as saying that even though SSN and II are incomparable, the former is "almost stronger" than the latter. The following proposition refers to the other side of the coin, by providing a sense in which II is "essentially weaker" than SSN.

Proposition 4. For each axiom $\alpha \in\{I R, T I N V, S . C O N T\} \equiv \mathcal{A}$ there exists a solution, $\mu^{\alpha}$, that satisfies II, violates SSN, and from the list $\mathcal{A}$ it violates only $\alpha$.

Proof. It is easy to see that for $\alpha=\mathrm{S}$.CONT one can take $\mu^{\alpha}=E_{l e x}$. It remains to show two solutions, each of which satisfies II and S.CONT, violates SSN, and violates exactly one axiom from $\{\mathrm{IR}, \mathrm{TINV}\}$. Here are two such solutions for $N=2$.

For each feasible set $S$, there is a maximal number $m$ such that $S \subset\left\{x \in \mathbb{R}^{2} \mid x \geq\right.$ $(m, m)\}$; next, there is a minimal $\epsilon \geq 0$ such that $(m+\epsilon, m+\epsilon) \in S$. Let $o(S)$, the 
origin of $S$, be defined by $o(S) \equiv(m+\epsilon, m+\epsilon)$. Consider the modified Nash solution, $\tilde{N}$, that picks the unique maximizer $x \in S$ of the product $\left(x_{1}-o_{1}(S)\right) \times\left(x_{2}-o_{2}(S)\right)$. It is easy to see that $\mu^{\alpha}=\tilde{N}$ works for $\alpha=$ IR.

Next, consider $\alpha=$ TINV. Let $q=\left(q_{1}, q_{2}\right)>\mathbf{0}$ be such that $q_{1} \neq q_{2}$ and consider

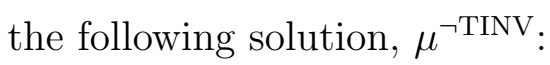

$$
\mu^{\neg \mathrm{TINV}}(S, d) \equiv \begin{cases}E(S, d) & \text { if } d \in \mathbb{Q}^{2} \\ \mu_{q}(S, d) & \text { if } d \notin \mathbb{Q}^{2}\end{cases}
$$

To see that $\mu\urcorner^{\text {TINV }}$ satisfies II, note that if $d \in \mathbb{Q}^{2}$, then every (arbitrarily small) rational $\varepsilon$ satisfies $d+(\varepsilon, \varepsilon) \in \mathbb{Q}^{2}$; if $d \notin \mathbb{Q}^{2}$, then we can find an arbitrarily small $\varepsilon>0$ such that $d^{\prime}=d+\varepsilon \cdot\left(1, \frac{q_{2}}{q_{1}}\right)$ is such that $d_{1}^{\prime} \notin \mathbb{Q}$ (because if such an $\varepsilon>0$ does not exists, it follows that there exists a continuum of rational numbers). To see that $\mu^{\urcorner \text {TINV }}$ violates SSN, consider $S \equiv\left\{x \in \mathbb{R}_{+}^{2} \mid x_{1}+x_{2} \leq \sqrt{2}\right\}$ and $T \equiv\left\{x \in \mathbb{R}_{+}^{2} \mid x_{1}+x_{2} \leq 5\right\}$. It is easy to see that $\left.\left.\mu\urcorner^{\operatorname{TINV}}(T,(0,0)) \neq \mu^{\neg \operatorname{TINV}}\left(T,\left(\frac{\sqrt{2}}{2}, \frac{\sqrt{2}}{2}\right)\right)=\mu\right\urcorner^{-\operatorname{TINV}}(T, \mu\urcorner^{-\operatorname{TINV}}(S,(0,0))\right)$. Finally, it is obvious that $\mu^{\neg \text { TINV }}$ satisfies IR and S.CONT, and violates TINV.

Acknowledgments I would like to thank Nejat Anbarci for helpful exchanges, an anonymous referee for helpful comments, and Ehud Kalai for many stimulating conversations.

\section{References}

Anbarci, N. (1993), Noncooperative foundations of the area monotonic solution, Quarterly Journal of Economics, 108, 245-258.

Anbarci, N. and Bigelow, J. (1994), The area monotonic solution to the cooperative bargaining problem, Mathematical Social Sciences, 28, 133-142.

Aumann, R. J. and Maschler, M. (1985), Game-theoretic analysis of a bankruptcy 
problem from the Talmud, Journal of Economic Theory, 36, 195-213.

Chun, Y. (1988), The equal-loss principle for bargaining problems, Economic Letters, 26, 103-106.

Chun, Y. (1989), Lexicographic egalitarian solution and uncertainty in the disagreement point, Mathematical Methods of Operations Research, 33, 259-266.

Chun, Y. and Thomson, W. (1990), Bargaining with uncertain disagreement point, Econometrica, 58, 951-959.

Kalai, E. (1977), Proportional solutions to bargaining situations: interpersonal utility comparisons, Econometrica 45, 1623-1630.

Kalai, E. and Smorodinsky, M. (1975), Other solutions to Nash's bargaining problem, Econometrica 43, 513-518.

Nash, J. F. (1950), The bargaining problem, Econometrica 18 , 155-162.

Perles, M. A. and Maschler, M. (1981), The super-additive solution for the Nash bargaining problem, International Journal of Game Theory, 10, 163-193.

Peters, H. (1986), Characterizations of bargaining solutions by properties of their status quo sets, Research Memorandum RM-86-012, University of Limburg, Maastricht.

Roth, A. (1977), Individual rationality and Nash's solution to the bargaining problem, Mathematics of Operations Research, 2, 64-65. 
Thomson, W. (1994), Cooperative models of bargaining, in R. J. Aumann and S. Hart (eds.) Handbook of game theory with economic applications, vol.2, Elsevier, New York. 